\title{
Inducible Myocardial Ischemia
}

National Cancer Institute

\section{Source}

National Cancer Institute. Inducible Myocardial Ischemia. NCI Thesaurus. Code C119207.

A finding of abnormal heart wall motion contractility in response to stress, and which is not seen at rest. 